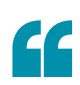

the clinical response to could be

predicted

by the composition

of the gut microbiome PD1 blockade

TUMOUR MICROENVIRONMENT

\section{Microbes matter}

Preclinical mouse models have shown that the gut microbiome can modulate therapeutic responses to cancer therapies. Yet, this has not been extensively characterized in humans. Two studies now propose that the gut microbiome is an important host factor that determines the response and primary resistance to anti-programmed cell death protein 1 (PD1) immunotherapy in patients with cancer.

Both groups initially sought to determine whether the clinical response to PD1 blockade could be predicted by the composition of the gut microbiome. To achieve this, faecal samples were collected from patients with either melanoma, non-small-cell lung cancer (NSCLC) or renal cell carcinoma (RCC) before and after commencement of immunotherapy. Metagenomic shotgun sequencing was then used to quantify bacterial species. A common finding was that high diversity of the gut microbiome correlated with prolonged progression-free survival (PFS) following PD1 inhibition.

Gopalakrishnan et al. evaluated patients with

CarlConway/Macmillan Publishers Limited

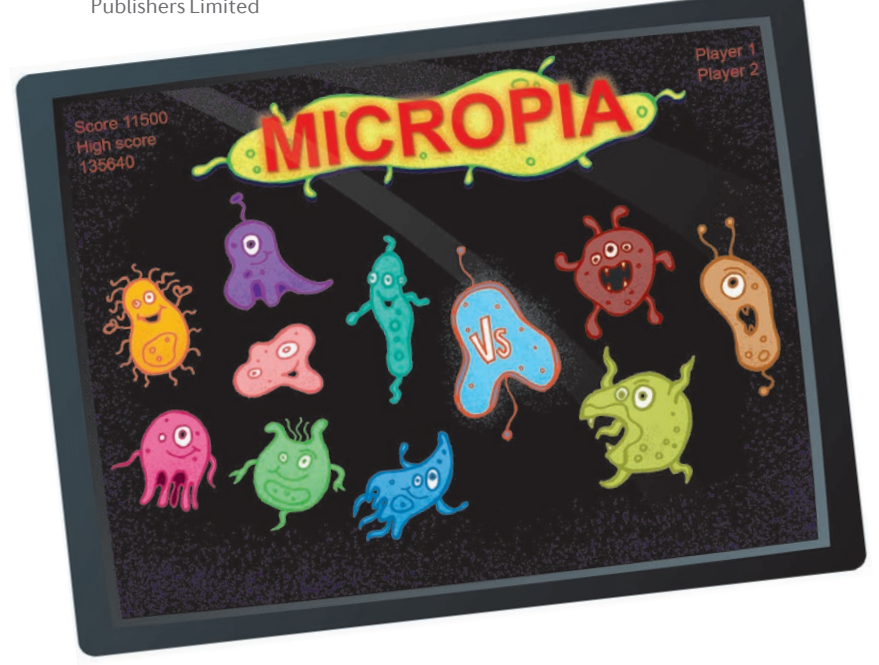

melanoma and found differentially abundant bacteria in the faecal microbiota, with Faecalibacterium enriched in responders to anti-PD1 therapy and Bacteroidales enriched in non-responders. Further analysis through functional genomic profiling of the gut microbiome samples revealed changes in metabolic functions, with anabolic pathways predominating in responders and catabolic pathways predominating in non-responders. Immune profiling of the tumour microenvironment (TME) showed that there was a positive correlation between the melanoma-associated $\mathrm{CD} 8^{+} \mathrm{T}$ cell infiltrate and the abundance of Faecalibacterium in responders.

Compositional differences in commensals were also associated with systemic immune responses; patients with an increased abundance of Faecalibacterium in the gut microbiota had an increased number of systemic effector $\mathrm{CD}^{+}$and $\mathrm{CD}^{+}$ $\mathrm{T}$ cells with a sustained cytokine response to $\mathrm{PD} 1$ inhibition, whereas patients with a greater abundance of Bacteroidales had an increased number of systemic regulatory $\mathrm{T}$ $\left(\mathrm{T}_{\text {reg }}\right)$ cells and myeloid-derived suppressor cells (MDSCs) with a dampened cytokine response. Interestingly, the authors noted that no differences were observed in the oral microbiome analysed from buccal samples of patients with melanoma.

Routy et al. assessed the gut microbiome of patients with NSCLC and RCC and found that an abundance of the bacterial species Akkermansia muciniphila was correlated with a positive clinical outcome to anti-PD1 therapy. Importantly, the authors also observed that antibiotic treatment, which can change the composition of the gut microbiota, negatively impacted the therapeutic efficacy of PD1 blockade.

To provide a mechanistic link between the dominance of specific bacterial species in the gut and favourable clinical responses, both groups performed faecal microbiota transplantation (FMT) experiments in germ-free mice. Gopalakrishnan et al. observed that mice who received patient stool from responders had decreased melanoma growth, increased levels of CD8 ${ }^{+}$ $\mathrm{T}$ cells and innate effector cells in the TME, and improved responses to PD1 blockade compared with those who received stool from non-responders. Routy et al. demonstrated that mice bearing MCA-205 sarcomas, receiving stool from patients with NSCLC who had responded to immunotherapy, had delayed tumour growth, sensitivity to PD1 blockade and a higher density of CXC-chemokine receptor $3(\mathrm{CXCR} 3)^{+} \mathrm{CD} 4^{+} \mathrm{T}$ cells, whereas those mice receiving stool from non-responders did not. Furthermore, these authors showed that recolonization of $A$. muciniphila in mice who had received stool from non-responders could rescue the impaired efficacy of anti-PD1 therapy through secretion of interleukin-12 (IL-12) from dendritic cells and increased infiltration of CC-chemokine receptor 9 (CCR9) ${ }^{+} \mathrm{CXCR}^{+} \mathrm{CD}^{+} \mathrm{T}$ cells into tumours.

Together, these findings point towards the possibility of therapeutically modulating the gut microbiome in patients with cancer to improve the success rate of immune checkpoint inhibitors.

Anna Dart

ORIGINAL ARTICLES Gopalakrishnan, V. et al. Gut microbiome modulates response to anti-PD-1 immunotherapy in melanoma patients. Science http://dx.doi.org/10.1126/science.aan4236 (2017) Routy, B. et al. Gut microbiome influences efficacy of PD-1-based immunotherapy against epithelial tumors. Science http://dx.doi.org/10.1126/ science.aan3706 (2017)

FURTHER READING Roy, S. \& Trinchieri, G.

Microbiota: a key orchestrator of cancer therapy. Nat. Rev. Cancer 17, 271-285 (2017) 